\title{
Evaluation of virtual reality therapy in augmenting the physical and cognitive rehabilitation of war veterans
}

\author{
B K Wiederhold ${ }^{1,2}$ and M D Wiederhold ${ }^{2}$ \\ ${ }^{1}$ Interactive Media Institute, 6160 Cornerstone Court, Suite 155, San Diego, CA 92121 \\ ${ }^{2}$ Virtual Reality Medical Center, 6160 Cornerstone Court, Suite 155, San Diego, CA 92121 \\ 1'bwiederhold@vrphobia.com \\ ${ }^{1}$ www.interactivemediainstitute.com, ${ }^{2}$ www.vrphobia.com
}

\begin{abstract}
War veterans with neuromuscularskeletal injury often require significant treatment and rehabilitation, straining health care resources. In a study funded by the Office of Naval Research (ONR) and the Defense Advanced Research Projects Agency (DARPA), the Virtual Reality Medical Center (VRMC) is applying virtual reality therapy to injured military personnel at the Naval Medical Center San Diego (NMCSD). The goal of this study is to investigate whether augmenting traditional rehabilitation with VR (in this case, off-the-shelf interactive video games) will enable a more rapid and complete rehabilitation. Because VR is interactive, and encourages patients to use their entire body to reach goals in the game, it is conceivable that it will make rehabilitation sessions more comfortable and entertaining. Participants consist of 20 veterans with upper arm and shoulder injuries (rotator cuff tear, shoulder impingement, bursitis) or amputation. The participants are divided into two groups $(\mathrm{n}=10)$ : an experimental group, which receives traditional rehabilitation augmented by virtual reality therapy, and a control group, which undergoes traditional rehabilitation. Participants will complete ten treatment sessions in their respective condition. Though the study has not been completed, preliminary results based on subjective questionnaires and functional capacity indicate that the experimental condition may elicit increased heart rate and respiration. Participants in this group also seem to enjoy the music and interaction made possible through VR. These results suggest that VR may enhance the rehabilitation process, creating a more effective form of treatment. Long-term benefits of this form of treatment may include improved treatment time and reduced drop out rates, therefore reducing the costs of rehabilitation.
\end{abstract}

\section{INTRODUCTION}

Rehabilitation is designed to restore a patient's physical, sensory, and/or mental capabilities lost due to injury, illness, or disease. Cognitive rehabilitation is a structured set of therapeutic activities designed to retrain an individual's ability to think, use judgment, and make decisions. Physical rehabilitation is the process of restoring a patient's lost function through hands-on treatment, exercise, and patient education. Cognitive and physical rehabilitation are among the largest sectors of healthcare costs in the United States. Total treatment expenditures are growing rapidly due to the aging of the United States population, as well as to higher rates of battle-related injury.

As of August 2005, it was estimated that over 12,500 U.S. warfighters had been wounded in Iraq. Because of advances in protective armor and battlefield medical care, more military personnel are surviving their wounds. This means that more people with amputated limbs, traumatic brain injury, and other injuries are returning to the U.S. for rehabilitation and treatment. The long term care of thousands of wounded veterans promises to be a large expense (Glasser, 2005).

This tremendous cost burden to the healthcare system generates an immediate need for new clinical approaches to both improve the efficacy of physical and cognitive rehabilitation and to reduce the total cost of such treatment. Recent improvements in computer and sensor technology now make it possible to develop portable home telerehabilitation systems that have the potential to dramatically improve rehabilitation 
outcomes for neurological and musculoskeletal injuries, while reducing overall rehabilitation costs by decreasing the need for in-person treatment (Hoenig, et al. 2006; Lewis, Deutsch, \& Burdea, 2006; Sugarman, Dayan, Weisel-Eichler, \& Tiran, 2006).

Aside from the obvious hurdle of expense, rehabilitation treatments are often less effective because they are unpleasant or monotonous. Duncan et al. (2002) found that compliance and adherence to rehabilitation therapy and guidelines are associated with improved treatment outcomes. While the focus of their research was stroke patients, it is not difficult to see how this principle would apply to other populations. Virtual Reality (VR) has the potential to help achieve the compliance necessary for effective rehabilitation. VR refers to computer-generated 3-D interactive synthetic environments, examples of which include video games. By increasing the motivation and enthusiasm for a patient's compliance with their physical rehabilitation therapy, VR becomes an advantageous addition to traditional physical therapy for patients.

For example, in a study with seven post-stroke elderly patients undergoing physical rehabilitation therapy for a weak arm, patients interacted with a virtual environment (VE) using a Sony PlayStation II EyeToy ${ }^{\mathrm{TM}}$. Researchers encouraged patients to use their weak arms when interacting with the games in the VE. By the end of the experiment, two chronic stroke patients had succeeded in using their weak arm to clean the left side of a virtual window. All of the patients reported that they had enjoyed the experience (Rand, Kizony, Weiss, 2004).

Other studies have supported this result. In a research study by Sveistrup et al. (2003), individuals presenting frozen shoulder due to musculoskeletal pathology went through exercise-training sessions, three times a week for six weeks, under three conditions: 1) conventional stretching, 2) traditional exercise, and 3) virtual reality exercise. Results indicated that virtual applications for rehabilitation increased the amount of interaction between patients and their environment. In 2001, Aaron, Rose, Janesen, and Hentz used a glovebased VR system for rehabilitation in patients with carpal tunnel syndrome and other hand-related injuries. Results showed that participants complied better with their rehabilitation program when they used a glovebased VR system than when they used traditional physical therapy alone. Other studies with stroke patients using VR therapy have shown improvements in arm movement and upper extremity functions (Piron, et al., 2003; Piron, et al., 2005; Holden, Dyar, Schwamm, \& Bizzi, 2005). In some patients, improvements in arm movement have also corresponded with improved scores on measurements of independence of ADL (Piron, et al., 2003; Piron, et al., 2005). VR has also been used to retrain upper limb motor movements, e.g., pouring water, in patients with acquired brain injury (Holden, Bettwiler, Dyar, Niemann, \& Bizzi, 2001).

The advantages of VR extend to cognitive rehabilitation as well. It can be used for vocational training and as a way to train cognitive tasks in brain damaged patients (Rose, Brooks, \& Rizzo, 2005). VR research in the field of motor rehabilitation includes applications for stroke, acquired brain injury, Parkinson's disease, orthopedic rehabilitation, balance training, wheelchair mobility, and training in functional activities of daily living (Holden, 2005). Research in VR applications for stroke patients in particular have made considerable progress; fMRI studies of VR used in conjunction with traditional physical therapy in stroke patients have resulted in neuroplastic changes in the brain and corresponding improvements in motor functions (Jang, et al., 2005). A study by Hofmann et al. (2003) asked ten patients suffering from mild to moderate Alzheimer's disease to move through a virtual environment that simulated pictures of the patient's typical surroundings, home or usual shopping route, and photographs of the patient at an earlier age. Results indicated that after three weeks of training, patients could perform the task more quickly and needed less help navigating the environment. In addition, eight out of the ten patients made fewer mistakes.

Furthermore, patients with phantom limb pain, a frequently debilitating phenomenon among amputees, have responded to the application of virtual reality technology. Professor Jonathan Cole of the Poole Hospital, University of Bournesmouth, and the University of Southampton in the United Kingdom, reported on the use of synthetic environments for the alleviation of phantom limb pain (Gallagher, 2004). The enhancement of missing-limb perception through the use of VR technology has been equally effective in alleviating phantom limb pain, while at the same time permitting a much greater perceptual range of absentlimb motion. Currently, a number of experimental studies are exploring virtual reality as an assessment tool for patients in the healthcare system. Virtual Reality and other advanced technologies may serve as an objectifying method to track performance improvements over the course of therapy. In light of the high number of amputees in the veteran population in the United States, this application might be especially relevant to military needs. 


\section{VIRTUAL REHABILITATION}

\section{$2.1 \quad$ Rationale}

There are many reasons why VR applications are so effective for rehabilitation. First, VR is an interactive, experiential medium. In the same way that children and teenagers intuitively grasp computers, VR users become directly engaged with the effects of the virtual experience. In addition, VR creates a safe setting where patients can explore and act without feeling threatened (Riva, 2005). Patients can make mistakes without fear of dangerous, real, or humiliating consequences (Standen \& Brown, 2005). Moreover, unlike human trainers, computers are infinitely patient and consistent (Standen \& Brown, 2005). In cognitive rehabilitation, VR can be manipulated in ways that the real world cannot. For example, VR can convey rules and abstract concepts without the use of language or symbols for patients with little or no grasp of language. Indeed, VR has been used to support individuals with intellectual disabilities by training them in many skills of independent living, such as grocery shopping, food preparation, orientation, road crossing, and vocational training (Standen \& Brown, 2005).

In motor rehabilitation, there are essentially three major advantages that VR offers over traditional therapy alone. First, VR creates a safe, controlled environment for repetitive practice, and repetitive practice is crucial in learning motor tasks. Second, VR provides immediate, real-time feedback about performance. Finally, because of its interactive nature, VR can increase motivation by making the experience fun (Holden 2005).

The current study utilizes an experimental study design in which one subset of patients (the control group) will receive traditional rehabilitation treatment, while another subset of patients (the experimental group) will receive traditional rehabilitation treatment augmented by virtual reality therapy.

Off-the-shelf kinesthetic video games, such as Sony's Eyetoy ${ }^{\mathrm{TM}}$, Dance Dance Revolution and Taiko Drum Master, will be utilized as virtual reality therapy. Kinesthetic video games focus on the player's body movements for input and control instead of a traditional joystick or controller. (See Figures 1 and 2.) For example, Sony's Eye Toy contains soccer, window washing, and dancing scenarios that require the user to use arm and body movements to block balls, eliminate spots on a window, or complete specific dance moves. Dance Dance Revolution utilizes a game pad and asks users to step on specific areas of the pad at certain times. Taiko Drum Master is played by hitting the drum peripheral in time with notes traveling across the screen. Sensors in the drum's surface and rim record the accuracy of each hit. Tasks increase in difficulty from single notes, to drum rolls, to different rhythms and speeds.

The body and arm movements required to play these games could encourage improvement in balance, strength and range of motion. Due to the nature of kinesthetic video games, it is conceivable to use them for physical therapy in order to make treatment sessions more comfortable and entertaining, in turn making them less tedious.
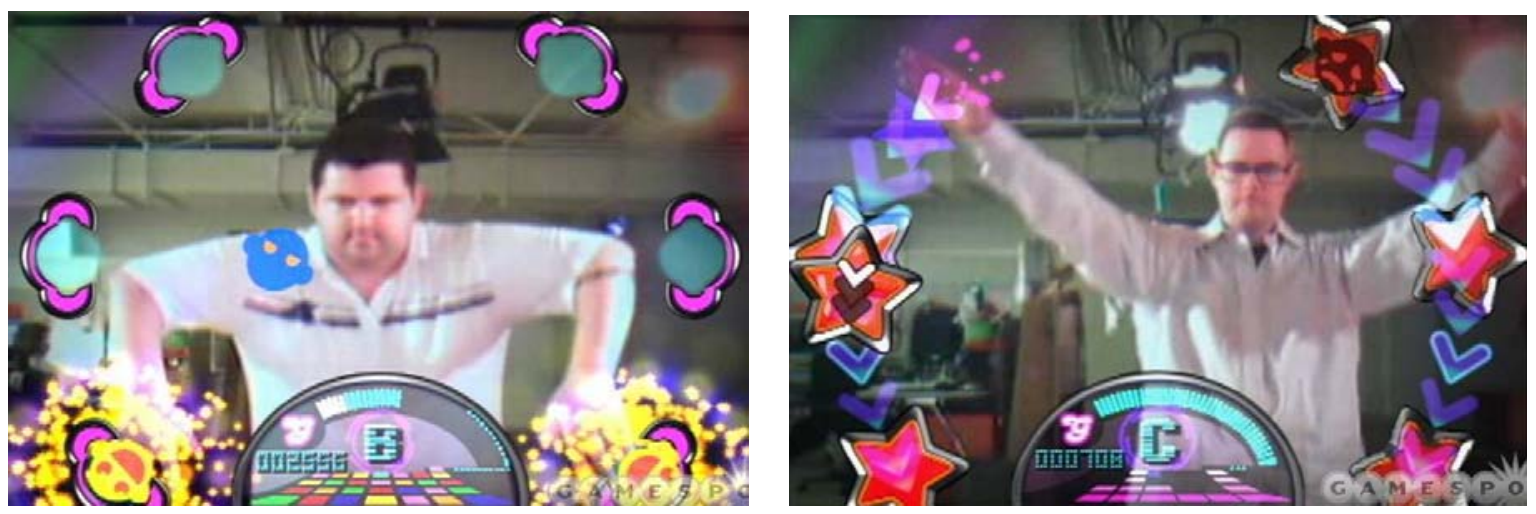

Figures 1 and 2. Screen shots from off-the-shelf video games utilizing Sony's Eyetoy ${ }^{\mathrm{TM}}$. Kinesthetic video games such as these focus on the player's body movements for input and control instead of a traditional joystick or controller.

The progress of patients in the control group and the experimental group will be measured using psychometric tests, physiological monitoring, and standardized physical therapy assessment tools. The outcomes of the control group will be compared to those of the experimental group. Based on existing research, the following hypotheses are posited for the current study: 
- H1. As compared to the control group, the experimental group will exhibit greater improvements in physical and psychological measures.

- H2. As compared to the control group, the experimental group will exhibit greater improvements in physical and psychological measures.

\subsection{Methodology}

2.2.1 Participants. The present study recruited 20 participants with arm amputations or upper arm and shoulder injuries (e.g. rotator cuff tear, shoulder impingement, bursitis). Participants were mixed in age, sex, and education, and were randomly assigned to the control group $(\mathrm{n}=10)$ or the experimental group $(\mathrm{n}=10)$. Each participant will complete ten sessions of treatment, taking part in either traditional rehabilitation activities (control condition) or traditional activities with the addition of VR (experimental condition). Participants endorsing current suicidal or homicidal ideation, meet diagnostic criteria for bipolar disorder, schizophrenia, or current substance dependence using the MINI were excluded from the study.

2.2.2 Materials. We are using off-the-shelf inexpensive video games to augment physical therapy and rehabilitation exercises. These inexpensive, interactive and entertaining tools are simple, but can serve as a platform to ask basic questions concerning patient and medical staff acceptance of this new format. We are conducting a clinical evaluation using this kinesthetic video game intervention to better understand the requirements necessary to develop and integrate a more tailored program that more specifically addresses the needs of patients undergoing therapy from injuries encountered in wartime.

2.2.3 Measures. Pre-test questionnaires include the Virtual Environment Pre-Treatment Questionnaire, the Beck Depression Inventory II (BDI-II), the Physical Therapy Evaluation Report, and the Overall Health Status Assessment. Participants will also complete a pre-participation physical evaluation administered by their physician. After therapy, the self-report questionnaires are completed again (BDI-II and Overall Health Status Assessment). In addition, participants fill out post-test questionnaires: an exit questionnaire, Virtual Environment Post-Treatment Questionnaire, and usability questionnaire. An evaluation will also be administered by the physical therapist. Follow up may be by phone or in person.

2.2.4 VR Scenario and Testing Procedure. Once participants complete all relevant pre-treatment consent forms and questionnaires, they are given an educational session to orient them to the equipment and methods used in the study. After the appropriate video game is chosen, participants were randomly assigned to one of two groups: the VR intervention group (those using the kinesthetic video games in addition to traditional physical therapy) or the control group (those experiencing traditional physical therapy treatment). Participants in both groups undergo physical therapy under direct supervision of their physical therapist and a research assistant who records subjective assessments.

In the experimental condition, participants complete traditional rehabilitation exercises and movements while using the kinesthetic video games. In these games, tasks progressively increase in difficulty. Participants are encouraged to attempt to earn a high score during each session with the games. At each session, participants' progress is monitored using psychometric tests, physiological monitoring, and standardized physical therapy assessment tools. After a full course of treatment, each participant completes the post-treatment questionnaires.

\section{RESULTS}

Though the current study is still underway, and therefore, firm results are not yet available, trends have begun to emerge during testing. As the study progresses, it appears that those in the experimental group (VR augmented rehabilitation) have an increased heart rate and respiratory rate during each session. In addition, motivation may be improved with the use of VR as subjective reports reveal that the participants enjoy the music and interaction provided by the VR.

\section{POTENTIAL IMPLICATIONS}

Due to the successful background in using simulation technology for the assessment and treatment of neuromuscular disorders, neural cognitive disorders and musculoskeletal injuries, there is a significant opportunity to apply these new treatment paradigms to those injured in the Iraq War. If the current study demonstrates, as hypothesized, that virtual reality therapy augments rehabilitation progress, the next step will be the development and application of more advanced rehabilitation technologies and strategies. That is, the current study has the potential to catalyze improvement and change within clinical rehabilitation at large. 
While these technologies will have immediate benefit for injured military personnel, their development will also serve to catalyze improvement and change within clinical rehabilitation at large. VR may indeed help create a more enjoyable and effective method of rehabilitating patients with war-induced injuries than the current paradigm.

Acknowledgements: The authors would like to acknowledge the Office of Naval Research (ONR) and the Defense Advanced Research Projects Agency (DARPA) for supporting this project.

\section{REFERENCES}

D H Aaron, B W Rose, C Janesen, V R Hentz, (2001). Pilot study of the Greenleaf Telerehabilitation System. Greenleaf Medical Telerehabilitation Executive Summary.

P W Duncan, R D Horner, D M Reker, G P Samsa, H Hoenig, B Hamilton, B J LaClair, T K Dudley (2002), Adherence to postacute rehabilitation guidelines is associated with functional recovery in stroke, Stroke, 33(1), pp. 167-77.

S Gallagher (2004), Nailing the lie: An interview with Jonathan Cole, Journal of Consciousness Studies, 11(2), pp. 3-21.

R J Glasser (2005), A ware of disabilities: Iraq's hidden costs are coming home, Harper's Magazine, http://uslaboragainstwar.org/article.php?id=8862.

H Hoenig, J A Sanford, T Butterfield, P C Griffiths, P Richardson and K Hargraves (2006), Development of a teletechnology protocol for in-home rehabilitation, Journal of Rehabilitation Research and Development, 43(2), pp. 287-298.

M Hofmann, A Rosler, W Schwarz, F Müller-Spahn, K Kräuchi, C Hock, et al (2003), Interactive computertraining as a therapeutic tool in Alzheimer's disease, Comprehensive Psychiatry, 44, pp. 213-219.

M K Holden, A Bettwiler, T Dyar, G Niemann, E Bizzi (2001), Retraining movement in patients with acquired brain injury using a virtual environment, Stud Health Technol Inform, 81, pp.192-8.

M K Holden, (2005), Virtual environments for motor rehabilitation: review, CyberPsychology \& Behavior, 8(3), pp.187-211.

M K Holden, TA Dyar, L Schwamm, E Bizzi (2005), Virtual-environment-based telerehabilitation in patients with stroke, Presence, 14(2), pp. 214-233.

S H Jang, S H You, M Hallett, Y W Cho, C M Park, S H Cho, HY Lee, T H Kim (2005), Cortical reorganization and associated functional motor recovery after virtual reality in patients with chronic stroke: an experimenter-blind preliminary study, Arch Phys Med Rehabil, 86, pp. 2218-23.

J A Lewis, J E Deutsch, G Burdea (2006), Usability of the remote console for virtual reality telerehabilitation: formative evaluation, CyberPsychology and Behavior, 9(2), pp. 42-147.

L Piron, P Tonin, AM Atzori, C Zucconi, C Massaro, E Trivello, M Dam (2003), The augmented-feedback rehabilitation technique facilitates the arm motor recovery in patients after a recent stroke, Stud Health Technol Inform, 94, pp. 265-267.

L Piron, P Tonin, F Piccione, V Iaia, E Trivello, M Dam (2005), Virtual environment training therapy for arm motor rehabilitation, Presence, 14(6), pp. 732-740.

D Rand, R Kizony, P L Weiss (2004), Virtual reality rehabilitation for all: Vivid GX versus Sony PlayStation II EyeToy, Proceedings of the 5th International Conference on Disabilities, Virtual Reality, \& Associated Technologies, Oxford, UK, Sept. 18-20, 2004, pp. 87-94.

G Riva G (2005), Virtual Reality in Psychotherapy: Review. CyberPsychology \& Behavior, 8(3) pp. 220230.

F D Rose, B M Brooks, A A Rizzo (2005), Virtual Reality in Brain Damage Rehabilitation: Review, CyberPsychology \& Behavior, 8(3), pp. 241-262.

P J Standen, D J Brown (2005), Virtual Reality in the Rehabilitation of People with Intellectual Disabilities: Review, CyberPsychology \& Behavior, 8(3), pp. 272-282.

H Sugarman, E Dayan, A Weisel-Eichler, J Tiran (2006), The Jerusalem TeleRehabilitation System, a new low-cost, haptic rehabilitation approach, CyberPsychology and Behavior, 9(2), pp. 178-182. 
H Sveistrup, J McComas, M Thornton, S Marshall, H Finestone, A McCormick, et al (2003), Experimental studies of virtual reality-delivered compared to conventional exercise programs for rehabilitation, Cyberpsychology \& Behavior, 6, pp. 245-249. 